\title{
A Comparative Study of Microorganisms Adhered to Different Surfaces of Temporary Removable Partial Dentures
}

\author{
Sharma $\mathrm{A}^{1^{*}}$, Shrestha $\mathrm{B}^{2}$, Chaudhari $\mathrm{BK}^{3}$, Singh RK ${ }^{4}$, Suwal $\mathrm{P}^{5}$ \\ ${ }^{1}$ Assistant Professor, Department of Prosthodontics, CODS, BPKIHS, Dharan, \\ ${ }^{2}$ Lecturer, Department of Prosthodontics, KDCRC, Kathmandu, \\ ${ }^{3}$ Consultant, Department of ENT, Bir Hospital, Kathmandu \\ ${ }^{4}$ Additional Professor, Department of Prosthodontics, CODS, BPKIHS, Dharan \\ ${ }^{5}$ Professor, Department of Prosthodontics, CODS, BPKIHS, Dharan
}

\begin{abstract}
Introduction: The purpose of this study was to identify and compare aerobic microorganisms adhering to polished and tissue surfaces of temporary removable partial dentures at $24 \mathrm{hrs}$ and at one week of dentures in use.

Materials and methods: A total of 31 patients visiting the department of Prosthodontics for temporary removable partial dentures without any known systemic and oral diseases were included in the study. Dentures were fabricated according to the standard prosthodontic procedures accepted in the department. Each patient was examined twice, once after $24 \mathrm{hrs}$ of denture insertion, and then at oneweek of use wherein plaque samples were collected from the polished surface and the tissue surface. The samples were taken to the microbiology lab for microbiological investigations within half an hour of sample collection and different microorganisms were identified.

Results: Microorganisms identified at 24 hours from polished and tissue surfaces of the dentures were same in type and not significantly different in frequency of isolation. They were Streptococcus spp., Staphylococcus spp., Acinetobacteranitratus, Enterobacter cloacae, Pseudomonas aeruginosa, Citrobacterfreundii and Candida albicans. Those identified at one week of dentures in use, from the two surfaces were same in type but three new types of microorganisms, Klebsiella pneumonia, Escherichia coli, and Proteus vulgaris were also found. There was a significant difference in the isolation frequency of Staphylococcus spp. from the two surfaces.

Conclusion: Same types of microorganisms adhere to both the surfaces but significant differences may occur in frequency of isolation. With time denturesfavour colonization of new microorganisms.
\end{abstract}

Key words: Adherence, denture microorganisms, denture plaque, surfaces of dentures, temporary removable partial denture

\section{Introduction}

Nynthetic acrylic resins used for fabrication of temporary removable partial dentures (RPDs)are susceptible to microbial adhesion, colonization and growth with time. RPDs can offer a reservoir for microorganisms. In the denture plaque, a variety of harmful

\footnotetext{
*Corresponding Author

Dr. Arati Sharma, Assistant Professor, Department of Prosthodontics; CODS, B P Koirala Institute of Health Sciences, Dharan, Nepal

E-mail: aratisharma122@yahoo.com
}

products may be produced by both the yeasts and the bacteria, which may provoke mucosal inflammation and once the mucosa is inflamed its barrier function against microbial products is diminished. ${ }^{1}$

Oral bacteria may be risk factors for a number of prevalent systemic diseases also ${ }^{2,3}$. Therefore, attention should be paid to the bacterial population in denture plaque. In addition to the significant gram-positive and fungal isolates, the gram-negative infections that become systemic are of particular concern ${ }^{4}$. 
A general concept is that microorganisms colonize tissue surface (TS) of the denture and polished surface (PS) being smooth is considered almost free of microorganisms. So, the purpose of this study was to know the aerobic microorganisms adhering to different surfaces of RPDs at $24 \mathrm{hrs}$ and at one week of dentures in use and to compare the type and frequency of microorganisms isolated from the two different surfaces.

\section{Materials and Methods}

A total of 31 patients visiting the department of prosthodontics, college of dental surgery (CODS), B.P. Koirala Institute of Health Sciences, Dharan, Nepal for RPD treatment agreed to participate in the study. Inclusion criteria were all the patients visiting the department of Prosthodontics for RPD except those with any known systemic disease, infectious disease, unhealthy oral mucosa, any history of antibiotic therapy for the last 3months, any adverse oral habits like smoking and patients not willing to give consent. RPDs were fabricated according to the standard prosthodontic procedures accepted in the department. Each patient was examined twice, once after $24 \mathrm{hrs}$ of RPD insertion, then after one-week of RPD in use. Ethical clearance was obtained from Institutional Ethical Review Board of BPKIHS, Dharan, Nepal.

\section{Sample collection}

RPD was taken out from the patient's mouth and the layer of saliva over it was washed-off with sterile normal saline. ${ }^{4}$ Sterile cotton $\mathrm{swab}^{2,5}$ stick was used to take the plaque sample by rotating the stick 5 times antero-posteriorly and mediolaterally respectively. ${ }^{4}$ Two plaque samples were collected from each patient. One plaque swab was taken from TS of the RPD adjacent to one of the abutment tooth and another plaque swab was taken from the PS adjacent to one of the abutment tooth.

\section{Transport of the sample}

Each swab sample was vortexed with hand in $2 \mathrm{ml}$ of normal saline in a sterile vial and taken to the microbiology lab for culture within half an hour of sample collection.

\section{Culture and colony counting}

Membrane Filter (Millipore; 0.45-micron meter, $047 \mathrm{~mm}$ ) was placed in a sterile petridish and the diluted sample was spread over the membrane filter. It was left to dry for some time and then transferred with a sterile forceps on the Blood Agar. All these procedures were performed in a disinfected area within 1- feet of a burning Bunsen burner. After incubation of $12 \mathrm{hrs}$, colony counting was done under the colony counter.

\section{Sub cultures}

Sub-cultures were performed repeatedly on Blood Agar and Mac Conkey Agar until pure bacterial isolates were obtained. Each representative colony was described according to its size, shape, margin, elevation, pigment, density and surface. Thus, differences in colony morphologies helped to some extent to identify microorganisms. Also, the type of hemolysis present or absent in the blood agar and presence or absence of Lactose fermentation in Mac Conkey agar helped to some extent to distinguish the microorganisms.

\section{Preparation of Smear and Gram-staining}

After describing the colony morphology, the microorganisms were further identified by their differences in cellular morphologies and staining properties. For this, smear was prepared and gram staining was done. Microscope slides were cleaned and dried thoroughly. The surface of the slide over which the smear was to be spread was flamed. The slide was labeled and a drop of sterile normal saline was put on it. A portion of colony from the culture plate was picked up 
with a sterile straight wire and emulsified with the normal saline and spread evenly. The smear was air dried, and then heat fixed by passing the dried slide three times slowly over the flame and allowed to cool. The inoculating wire was flamed and reflamed each time before picking up a portion of a colony. All these procedures were performed in a disinfected area within 1 -feet of a burning Bunsen burner.

The heat fixed smear was covered with crystal violet stain for one minute. Then, the stain was rapidly washed off with clean water. Remaining water on the slide was tipped off and the smear was covered with Lugol's Iodine for one minute. Iodine was washed off with clean water. Then, decolourisation was done rapidly with Acetone and the smear was washed immediately with clean water. The counter stain was done with $0.1 \%$ Carbol-Fuchsin for 30 seconds. The stain was washed off with clean water. The smear was air dried and examined under oil immersion using a compound microscope. Findings were recorded. Thus, the microorganisms were identified as either gram positive or gram negative and either coccus-shaped or rodshaped.

\section{Biochemical tests}

To aid in the more definitive identification of the microorganisms, a series of biochemical tests were performed like Catalase Test, Coagulase Test, Triple Sugar Iron Test, Sulphide Indole Motility Test, Urease Test and Citrate Test.

All the data collected were entered in Microsoft Excel and data analysis was done using SPSS version 10 (SPSS, Inc., Chicago, IL). McNemar's test was applied.

\section{Results}

Results obtained from the 31 temporary removable partial dentures at $24 \mathrm{hrs}$

The colony count obtained were $\leq 200$ from all the samples taken from PS and 27 samples of
TS. 4 samples showed colony count of $\geq 300$ from TS.

Sub-cultures obtained a total of 56 isolates from PS and 51 isolates from TS. Among the 56 isolates from the PS, 48 were Gram positive cocci (GPC); 7 were Gram negative bacilli (GNB) and 1 isolate was Candida albicans. Among the 51 isolates obtained from the TS, 43 isolates were GPC; 7 isolates were GNB and 1 isolate was Candida albicans.

\section{Results obtained from the 31 temporary removable partial dentures at 1 week}

The colony counts obtained from the 31 dentures were $\leq 200$ counts from all the samples of PS and from TS, 2 samples showed colony count $\leq 200$ and remaining 29-samples showed colony count $\geq 300$.

Sub-cultures obtained a total of 88 isolates from the PS and 75 isolates from the TS. Among the 88 isolates of the PS, 54 isolates were GPC, 30 were GNB and 4 were Candida albicans.

Among the 75 isolates of the TS, 41 were GPC, 30 were GNB and 4 were Candida albicans. Staphylococcus species were isolated in significantly higher number $(\mathrm{p}<0.001)$ from PS compared to TS.

\section{Discussion}

Types of microorganisms isolated from the PS and from the TS were the same at $24 \mathrm{hrs}$. They were Streptococcus spp., Staphylococcus spp., Acinetobacteranitratus, Enterobacter cloacae, Pseudomonas aeruginosa, Citrobacterfreundii and Candida. Difference in the frequencies of isolation of microorganisms between the two surfaces was not significant at $24 \mathrm{hrs}$.

Three new types of microorganisms were isolated at 1 week in addition to those isolated at $24 \mathrm{hrs}$ from both the surfaces. Those microorganisms were Klebsiella pneumonia, Escherichia coli, and Proteus vulgaris. There was a significant difference in the frequency of 
Table 1: Microorganisms Identified at 24 hours from polished and tissue surfaces of RPDs

\begin{tabular}{|l|c|c|c|}
\hline Micro-organisms isolated & \multicolumn{2}{|c|}{ Number of Samples } & p value* \\
\hline Streptococcus spp. & Polished surface & Tissue surface & \\
\hline Staphylococcus spp. & 31 & 31 & 1.000 \\
\hline Acinetobacteranitratus & 17 & 12 & 0.125 \\
\hline Enterobacter cloacae & 3 & 3 & 1.000 \\
\hline Pseudomonas aeruginosa & 2 & 2 & 1.000 \\
\hline Citrobacterfreundii & 1 & 1 & 1.000 \\
\hline Candida albicans & 1 & 1 & 1.000 \\
\hline
\end{tabular}

* McNemar's test

Table 2: Microorganisms Identified from polished and tissue surfacesat 1 week of RPD in use.

\begin{tabular}{|l|c|c|c|}
\hline Micro-organisms isolated & \multicolumn{2}{|c|}{ Number of Samples } & p value* \\
\hline Streptococcus spp. & Polished surface & Tissue surface & \\
\hline Staphylococcus spp. & 31 & 31 & 1.000 \\
\hline Klebsiellapneumoniae & 23 & 10 & 0.001 \\
\hline Acinetobacteranitratus & 8 & 8 & 1.000 \\
\hline Enterobacter cloacae & 5 & 5 & 1.000 \\
\hline Pseudomonas aeruginosa & 5 & 4 & 1.000 \\
\hline Escherichia coli & 3 & 5 & 0.625 \\
\hline Proteus vulgaris & 2 & 1 & 1.000 \\
\hline Citrobacterfreundii & 4 & 4 & 1.000 \\
\hline Candida albicans & 3 & 3 & 1.000 \\
\hline
\end{tabular}

* McNemar's test

isolation of Staphylococcus spp. from the PS as compared to the TS. Differences in the frequency of isolation of other microorganisms from the two different surfaces were not significant.

Streptococcus spp. was a constant finding from all the samples both at $24 \mathrm{hrs}$ and at 1 -week of RPD in use. The second most commonly isolated microorganisms were Staphylococcus spp. The frequency of isolation of GNB was low at $24 \mathrm{hrs}$ that increased significantly at 1 week.

S D Harding et al (1991) ${ }^{4}$ conducted a study to see the Cultivable microflora of denture plaque from patients with denture-induced stomatitis and they also found that Streptococcus spp. were present in all of the samples and Staphylococcus aureus were yielded from 15\% of the samples cultured aerobically. But, in their study anaerobic culture was also done and the predominant cultivable microorganisms were an aerobic microorganisms. This was the main limitation of our study that we cultured only aerobic microorganisms. Another important difference was that in their study patients included were those suffering from denture induced stomatitis where as in our study patients were healthy.In contrast, Ohman S C et al. (1995) found that Staphylococcus aureus were isolated from only few cases and they concluded that despite frequent general diseases and medications, there is a low prevalence of Staphylococcus aureus in the denture plaque. Staphylococcus aureusis a major pathogen of increasing importance due to the rise in antibiotic resistance and our study has shown that polished surface of RPD can be a constant source of Staphylococcal infection and should be paid attention. 
Goldberg S et al. (1997) ${ }^{10}$ found very high prevalence of Enterobacteriaceae like Klebsiella and Enterobacter in the oral cavities of the denture-wearing population as compared with the other groups. In contrast Ohman S C et al(1995) ${ }^{9}$ found the bacteria of Enterobacteriacae family in a few cases only in their study. In our study, the frequency of isolation of Enterobacteriacae (GNB) was very low at $24 \mathrm{hrs}$ but increased significantly at 1 week of dentures in use. Several researchers have studied interactions among Candida and bacteria in an attempt to determine how oral bacteria may modulate Candida adherence and colonization and have found that with the exception of the glycocalyx producer $P$. aeruginosa, the preformed biofilms of bacteria significantly reduced adhesion and biofilm growth of C. albicans ${ }^{12,13}$.

Further work is required on the cell walls of the micro organisms to determine the specific adhesins and how these complex molecules relate to the salivary pellicle that forms the surfaces at different denture base materials. The medical community has placed little importance on daily care in denture wearers for the prevention of systemic infections. ${ }^{2}$ Therefore, while diagnosing oral or systemic infections in an elderly RPD wearing patient, physician must pay attention to denture plaque microorganisms so that life threatening infections can be prevented. Knowledge of the types of microorganisms adhering to different surfaces of RPDs can also be used to evaluate the efficacy of different denture cleansers in preventing colonization of the RPD by these pathogenic microorganisms. ${ }^{14-17}$

\section{Conclusion}

Microorganisms isolated from PS and TS were same at $24 \mathrm{hrs}$ and there was no significant difference between the frequencies of isolation from the two surfaces. Three new types of microorganisms, Klebsiella pneumonia, Escherichia coli, and Proteus vulgaris were isolated at 1 week in addition to those isolated at $24 \mathrm{hrs}$ from both the surfaces. There was a significant difference in the frequencies of isolation of Staphylococcus spp. from the two surfaces at 1week. Streptococcus spp. was a constant finding from all the samples both at $24 \mathrm{hrs}$ and at 1-week of RPDs in use. The second most commonly isolated microorganisms were Staphylococcus spp. The frequency of isolation of GNB was low at $24 \mathrm{hrs}$ that increased significantly at 1 week andthe most favoured among GNB with time was Klebsiellapneumonae. PS also favour adherence of microorganisms and differences do occur between PS and TS. So, PS should not be neglected while cleaning dentures.

\section{References}

1. Jorgensen E B. Ecology of Candida-associated Denture Stomatitis. Microb Ecol Health Dis 2000;12: 170-85.

2. Sumi Y, Kagami H, Ohtsuka Y, six authors name needs to be mentioned, if more than six then et al.et al. High correlation between the bacterial species in denture plaque and pharyngeal microflora. Gerodontology 2003;20: 84-87.

3. Coulthwaite L VJ. Potential pathogenic aspects of denture plaque. Br J Biomed Sci 2007; 64: $180-9$.

4. S.D. last name followed by initials of first nameHarding, M. Wilson, C. Dickison, J. Howlett JH. The Cultivable Microflora of Denture Plaque from Patients with Dentureinduced stomatitis. Microb Ecol Health Dis 1991;4: 149-57.

5. Koopmans AS, Kippuw N de GJ. Bacterial Involment in Denture- induced stomatitis. $J$ Dent Resyear is not mentioned here67: 124650.

6. Fatma Alzahraa M. Gomaa ZHH. Isolation and Identification of Microorganisms Associated With Removable Denture: Prevalence of Non Oral Pathogens. Egypt Acad J Biol Sci 2010;2(2)75-82. 
7. P author's name???M. Presence of microorganisms on the fitting denture complete surface: study in vivo. J Oral Rehabil 2000;27: 708-13.

8. Sousa C, Teixeira P, Oliveira R. Influence of Surface Properties on the Adhesion of Staphylococcus epidermidis to Acrylic and Silicone. Int J Biomater 2009;2009: 1-9.

9. Ohman SC, Osterberg T, Dahlen G,et al only after six authors. The prevalence of Staphylococcus aureus, Enterobacteriaceae species, and Candida species and their relation to oral mucosal lesions in a group of 79-yearolds in Goteborg. Acta OdontolScand 1995;53: 49-54.

10. Goldberg S, Cardash H, Browning H 3rd, Sahly H RM. Isolation of Enterobacteriaceae from the mouth and potential association with malodor. $J$ Dent Res 1997;76: 1770-5.

11. Conti S, dos Santos SS, Koga-Ito CY JA. Enterobacteriaceae and Pseudomonadaceae on the dorsum of the Human Tongue. J Appl Oral Sci 2009; 17: 375-80.

12. Pereira-Cenci T, Del Bel Cury AA, Crielaard W TCJ. Development of Candida-associated denture Stomatitis: new insight. J Appl Oral Sci 2008; 16(2)86-94.

13. Bagg J, Silverwood RW. Coagglutination reactions between Candida albicans and oral bacteria. J Med Microbiol 1986;22: 165-169.

14. Paranhos Hde F, Panzeri H, Lara EH, Candido RC II. Capacity of Denture Plaque/Biofilm removal and Antimicrobial action of a new denture paste. Braz Dent J 2000;11: 97-104.

15. Gornitsky M,ParadisI I, Landaverde G, et al only after six authors. A clinical and microbiological evaluation of denture cleansers for geriatric patients in long-term care institutions. $J$ Can Dent Assoc 2002;68: 39-45.

16. da Silva FC, Kimpara ET, Mancini MN, Balducci I, Jorge AO K-IC. Effectiveness of six different disinfectants on removing Five microbial species and effects on the topographic characteristics of Acrylic resin. Journal of Prosthodontics. J Prosthodont 2008;17: 62733.

17. Dovigo LN, Pavarina AC, Ribeiro DG, et al only after six authors. Microwave disinfection of complete dentures contaminated in vitro with selected bacteria. J Prosthodont 2009;18: 611617. 